\title{
A rare case of scimitar syndrome with horseshoe lung
}

Scimitar syndrome is a rarely seen congenital cardiopulmonary anomaly characterised by partial or complete anomalous pulmonary venous drainage into the inferior vena cava via an anomalous descending vein that runs parallel to the right border of the heart giving an appearance of a curved Turkish sword or scimitar on plain chest radiography. Clinicians should also be aware of other interesting cardiopulmonary anomalies that can be seen in association with scimitar syndrome.

A 41-year-old female presented to the Chest Medicine Clinic (University of Oklahoma Health Sciences Center, Oklahoma City, OK, USA) with lifelong worsening dyspnoea. As part of the workup for her dyspnoea, a contrast-enhanced computed tomography (CT) scan of the chest was obtained. Coronal reconstruction of the CT scan showed a scimitar shaped vein running from the middle of the right lung towards the diaphragm and draining into the inferior vena cava (fig. 1a). Also visible was an anomalous midline pulmonary parenchymal isthmus fusing the right lower lobe and left lower lobe of the lungs posterior to the heart (fig. $1 \mathrm{~b}$ and c). Some degree of hypoplasia of the right lung was also seen on the CT scan (fig. 1). Based on these imaging results, a diagnosis of scimitar syndrome with horseshoe lung was made.

First described by COOPER [1] in 1836, scimitar syndrome is a rare congenital anomaly characterised by partial or complete anomalous pulmonary venous drainage of the right or left lung into the inferior vena cava. The term "scimitar" refers to the resemblance of the anomalous draining vein to a Turkish sword on
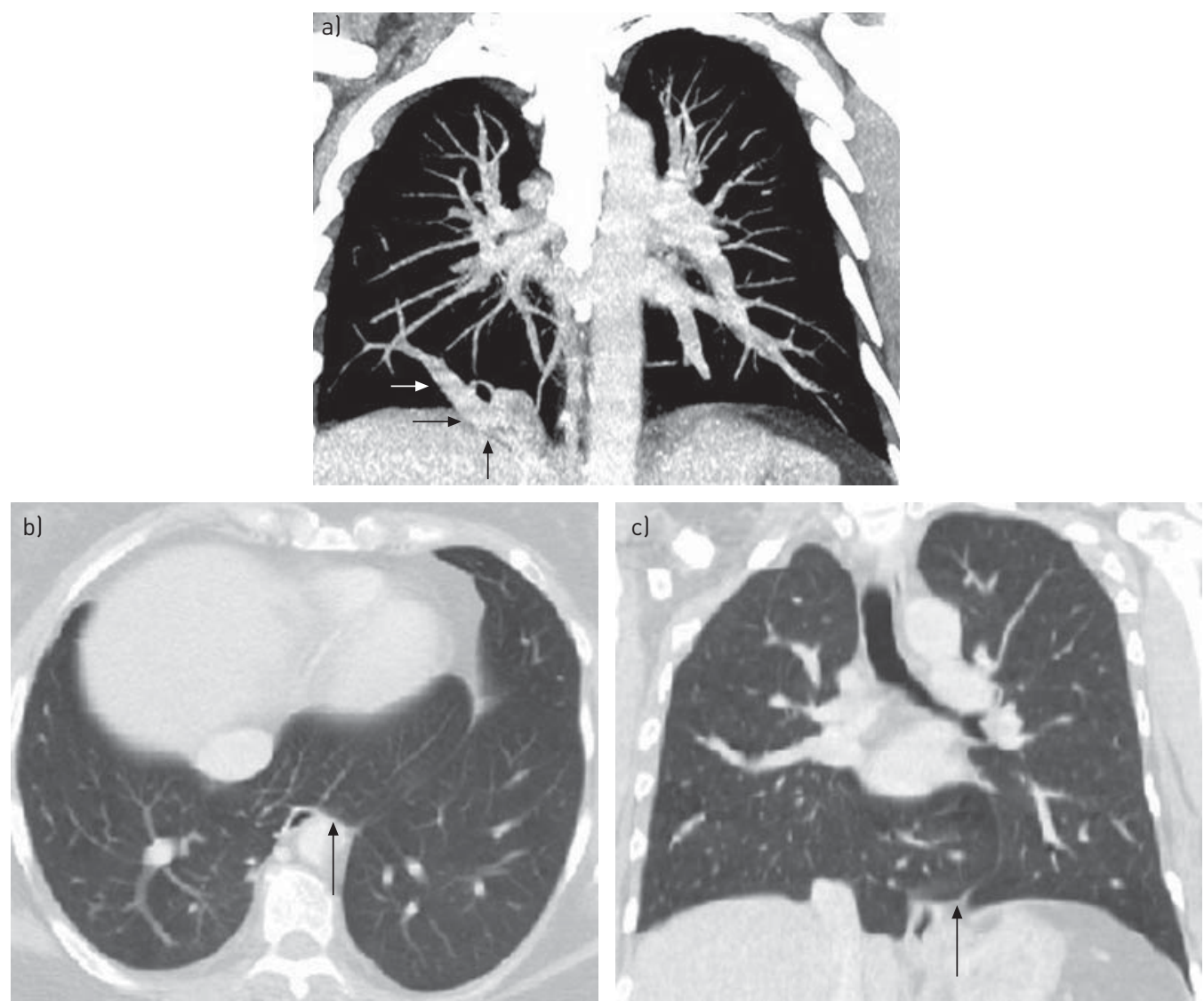

FIGURE 1 a) Contrast enhanced computed tomography (CT) scan (coronal reconstruction) showing anomalous right pulmonary vein (arrows). b) Axial CT scan showing horseshoe lung as demonstrated by the parenchymal isthmus connecting the right lung with the left lung (arrow). c) Coronal reconstruction CT scan demonstrating similar findings of horseshoe lung (arrow). In all images, hypoplasia of the right lung is seen with smaller right lung size compared to the left. 
plain chest radiography. The estimated incidence of this disorder is approximately two per 100000 births, with a 2:1 female preponderance. Although various other congenital cardiopulmonary abnormalities such as hypoplasia of the right lung, pulmonary sequestration, persisting left superior vena cava, various congenital intracardiac defects and dextroposition of the heart have been described in association with scimitar syndrome, horseshoe lung anomaly with scimitar syndrome is a very rare association [2]. The term "horseshoe lung" denotes an anomalous isthmus of pulmonary parenchyma extending from the base of the right lung and fusing with the base of the left lung (fig. $1 \mathrm{~b}$ and c). Up to $80 \%$ of reported cases of horseshoe lung have been seen in association with scimitar syndrome [3].

0 @ERSpublications

Clinicians should be aware of scimitar syndrome and cardiopulmonary anomalies as important causes of exertional dyspnoea http://ow.ly/qeOoI

Himanshu Bhardwaj ${ }^{1}$ and Bhaskar Bhardwaj ${ }^{2}$

${ }^{1}$ Dept of Pulmonary Medicine and Critical Care, University of Oklahoma Health Sciences Center, Oklahoma City, OK, and ${ }^{2}$ Heart Rhythm Institute, University of Oklahoma Health Sciences Center, Oklahoma City, OK, USA.

Correspondence: H. Bhardwaj, Dept of Pulmonary Medicine and Critical Care, University of Oklahoma Health Sciences Center, PO Box 26901, WP1310, Oklahoma City, OK 73190, USA. E-mail: himanshu-bhardwaj@ouhsc.edu

Received: July 312013 | Accepted after revision: Aug 242013

Conflict of interest: None declared.

Provenance: Submitted article, peer reviewed.

\section{References}

1 Cooper G. Case of malformation of the thoracic viscera consisting of imperfect development of the right lung and transposition of the heart. Lond Med Gaz 1836; 18: 600-601.

2 Hassberg D, Steil E, Sieverding L, et al. Combination of scimitar syndrome and horseshoe lung. A rare but typical finding - case report and review of the literature. Klin Padiatr 1992; 204: 434-443.

3 Takahashi M, Murata K, Yamori M, et al. Horseshoe lung: demonstration by electron-beam CT. Br J Radiol 1997; 70: 964 . 